\title{
Apolipoprotein B-48 as a marker for chylomicrons and their remnants: studies in the postprandial state
}

\author{
BY S. GAIL ISHERWOOD*, CHRISTINE M. WLLIAMS † AND BARRY J. GOULD \\ Nutrition Research Group, University of Surrey, Guildford GU2 5XH
}

Cardiovascular disease has become one of the major causes of death in Western countries. The role of endogenous lipoproteins in the process of atherogenesis is now well established, but the role of plasma triacylglycerol (TAG), especially in the postprandial state, has been largely overlooked until more recently. It was first proposed in 1979 that remnant particles of dietary-derived lipoproteins are atherogenic (Zilversmit, 1979). Studies have since shown that there is a link between coronary artery disease (CAD) and high magnitude and duration of postprandial lipaemia (Simpson et al. 1990; Groot et al. 1991). In light of these findings it is important to investigate any factors which may decrease postprandial lipaemia. Various methods have been used to characterize postprandial lipaemia, with the use of apolipoprotein (apo) B-48 as a marker for intestinally-derived lipoproteins being the most successful to date. The aim of the present review is to examine the techniques currently used to study postprandial lipoprotein particles and to describe some of the studies which have been carried out to investigate the effects of lifestyle factors, particularly exercise and diet, on postprandial lipaemia.

\section{METABOLISM OF CHYLOMICRONS}

TAG-containing chylomicrons (CM) are synthesized in the enterocyte in response to an influx of dietary fat. The CM core contains dietary TAG and cholesteryl ester (CE) and on the surface are cholesterol, phospholipids and the apolipoproteins, including apo B-48 the transport protein unique to intestinally-derived lipoproteins.

CM catabolism occurs as a two-step process. TAG is hydrolysed by the enzyme lipoprotein lipase (EC 3.1.1.34; LPL) in extra-hepatic tissues to produce non-esterified fatty acids which are taken up by these tissues. The resultant $\mathrm{CM}$ remnant (CMR) with its depleted TAG core is taken up by receptors in the liver. During its residence time in the circulation the CMR may interact with endogenous lipoproteins through the action of $\mathrm{CE}$ transfer protein (CETP), which transfers CE from HDL to CMR, and TAG from CMR to the HDL (Tall, 1993).

\section{THE LINK BETWEEN CORONARY ARTERY DISEASE AND POSTPRANDIAL LIPAEMIA}

Various studies have implicated postprandial TAG-rich lipoproteins (TGRL) and their remnants in the pathogenesis of CAD. Zilversmit (1979) proposed that CMR could be directly atherogenic and infiltrate the arterial endothelium in a similar manner to LDL and eventually lead to atherogenesis. Evidence in support of this theory has shown that CMR can be taken up into macrophages leading to the production of foam cells (Floren et al.

\footnotetext{
*Present address: Department of Biology, PO Box 373, University of York, York YO1 5YW. †Present address: Department of Food Science and Technology, University of Reading, Whiteknights, Reading RG6 6AP.
} 
1981; Chung \& Segrest, 1990). Two more recent studies have shown that TGRL can be taken up into arterial walls (Mamo \& Wheeler, 1994) and are found in atherosclerotic plaques (Rapp et al. 1994).

In contrast to the 'Zilversmit (1979) theory', Patsch and his group (Miesenbock \& Patsch, 1991) proposed that CMR were not themselves atherogenic particles, but that their interactions with other endogenous lipoproteins created an atherogenic state. According to this theory, a prolonged residence time of CMR in the circulation could lead to TAGenrichment of HDL through the action of CETP, thus increasing the metabolism and uptake of HDL and reducing the level of these so-called 'anti-atherogenic' lipoproteins.

Several clinical studies have provided evidence for the involvement of postprandial lipaemia in the risk of CAD. Patients with CAD were shown to have a higher magnitude and duration of postprandial lipaemia than healthy controls (Simpson et al. 1990; Groot et al. 1991; Ginsberg et al. 1995). Interestingly, a study of healthy young men who had a family history of CAD showed that these individuals had a higher postprandial lipaemia than the sons of healthy men (Uiterwaal et al. 1994).

\section{MARKERS FOR THE MEASUREMENT OF POSTPRANDIAL LIPOPROTEINS}

Three markers have been used to investigate postprandial lipoprotein metabolism: TAG, retinyl esters (RE) and apo B-48.

\section{Triacylglycerols}

The easiest method used to follow postprandial lipaemia is to measure the change in TAG concentration in blood samples taken for a period of hours after a meal (Patsch et al. 1983). However, the drawback with this approach is that there is no discrimination between endogenous VLDL-TAG and dietary-derived TAG in CM. In an attempt to look more selectively at CM, a TGRL fraction can be produced by ultracentrifugation (Weintraub et al. 1987). However, a pure CM fraction cannot be produced since the densities of CM and VLDL particles overlap.

\section{Retinyl esters}

Several studies of postprandial lipaemia have used exogenously-administered RE as a marker for dietary-derived lipoproteins, since the RE should remain in the CMR core until uptake by the liver (Weintraub et al. 1987; Berr, 1992). However, there are some concerns as to the validity of RE as a marker for intestinally-derived lipoproteins (Krasinski et al. 1990; Cohn et al. 1993; Blaner et al. 1994) and taken together these findings suggest that the level of RE may not specifically indicate the level of CM and CMR in the circulation.

\section{Apolipoprotein B-48}

The most obvious discriminating feature between lipoproteins of hepatic and dietary origin is the form of apo B on these particles. Apo B-48 is produced only in the intestine of human subjects and, therefore, is found only in lipoproteins carrying dietary lipids: CM and CMR. As there is only one apo B-48 per CM (Young, 1990) and no transfer of apo B-48 to other lipoproteins, the concentration of apo B-48 should give an accurate measure of CM and CMR levels. However, despite its potential importance, analysis of apo B-48 as a marker for dietary-derived lipoproteins has been limited since the protein is difficult to measure by 
conventional methods. The main problem is that the amino acid sequence of apo B-48 is identical to the N-terminal sequence of apo B-100; this has prevented antibodies from being raised specifically to apo B-48. In addition, the concentration of apo B-48 in the circulation is very low; in plasma it is $1-5 \mu \mathrm{g} / \mathrm{ml}$, which is between 100 - and 1000 -fold lower than the concentration of apo B-100 (Karpe \& Hamsten, 1994).

The most reliable and successful method to date for measuring apo B-48 has been electrophoresis of proteins on SDS-PAGE followed by staining of protein bands with Coomassie Blue and densitometry (Karpe \& Hamsten, 1994; Kotite et al. 1995). This was the method used originally to identify the two forms of apo B in plasma (Kane et al. 1980). However, the technique is long and complicated, the limit of detection is relatively high and very few samples can be measured in $1 \mathrm{~d}$, making it of limited use for large postprandial studies.

A breakthrough in the measurement of apo B-48 was made by raising a specific antibody to the protein (Peel et al. 1992). The antiserum exploited the fact that the Cterminal isoleucine residue of apo B-48 was negatively charged, unlike the equivalent isoleucine in apo B-100 where it is part of a peptide bond. In apo B-100 this is in a hydrophobic region of the protein which is probably buried in the lipoprotein particle, but in CM this negative charge on apo B-48 is accessible to the antibodies. This antiserum was originally used in a semi-quantitative immunoblotting technique (Peel et al. 1993). A more sensitive, quantitative ELISA assay has since been developed (Lovegrove et al. 1996) which has applications in large-scale clinical and dietary studies (Zampelas et al. 1995; Jackson et al. 1996).

\section{STUDIES OF APOLIPOPROTEIN B-48}

The use of the analytical SDS-PAGE method has allowed the investigation of the contribution of liver and intestine to postprandial lipaemia. Studies by both Cohn et al. (1993) and Schneeman et al. (1993) suggested that $80 \%$ of the postprandial increase in TAG is carried in CM particles. But, although postprandially there was a 5-6-fold increase in the apo B-48 level, $80 \%$ of the absolute increase in particle number was accounted for by VLDL particles. This reflects the very low concentration of apo B-48 in plasma and implies that relatively few CM carry the bulk of the TAG postprandially. A possible reason for the increase in the VLDL levels postprandially is competition for LPL and preferential hydrolysis of CM (Karpe \& Hultin, 1995).

Karpe and his group (Karpe et al. 1994; Karpe \& Hamsten, 1994) have investigated the metabolism of TGRL by studying apo B-48 and apo B-100 concentrations in four lipoprotein subfractions isolated by ultracentrifugation (Svedberg flotation unit (Sf); $>400$ large VLDL, 60-400 small VLDL, 20-60 IDL, 12-20 LDL). Karpe \& Hamsten (1994) showed that in normolipidaemic subjects in the fasting state the majority of apo B-48 was found in the Sf 20-60 fraction. Postprandially there was an increase in apo B-48 levels in all fractions except the Sf 12-20. However, because the level of apo B-48 in this Sf 12-20 fraction was on the limit of detection, this result may not be entirely accurate. In a study of young male post-infarction patients, Karpe et al. (1994) showed a significant correlation between levels of apo B-48 in the Sf 20-60 range $4 \mathrm{~h}$ after a meal (i.e. small CMR) and progression of CAD.

In their studies Karpe and his group (Karpe et al. 1994; Karpe \& Hamsten, 1994) found no evidence for the presence of apo B-48 in the small, dense lipoprotein size range, i.e. $\mathrm{Sf}<12$, in which the LDL particles would be isolated. Using the more sensitive immunoblotting technique, we have shown that apo B-48 is present in both the IDL and 
LDL subfractions isolated by ultracentrifugation (Isherwood et al. 1996). This finding suggests that CMR may be delipidated to such an extent before their removal by the liver, that they reach a size and density equivalent to that of IDL and LDL. This finding challenges current thinking regarding the distribution of dietary-derived lipoproteins and requires further investigation to determine its true significance.

\section{EFFECTS OF LIFESTYLE FACTORS ON POSTPRANDIAL LIPAEMIA}

\section{Effects of exercise}

Cross-sectional studies have established that athletes have a lower postprandial lipaemia than sedentary individuals, as indicated by lower peak TAG levels and lower areas under the curve (AUC) of TAG concentration over time (Merrill et al. 1989; Podl et al. 1994). This positive effect of exercise persists even when both the active and inactive subjects are matched for fasting TAG concentrations (Cohen et al. 1989). Longitudinal studies have also shown that exercise training improves the lipaemic response to a high-fat test meal (Weintraub et al. 1989; Mankowitz et al. 1992). In both these studies, postprandial CM and CMR levels were specifically monitored using retinyl palmitate (RP) as marker and each reported a significant effect of exercise on lipoprotein levels in the CM fraction and a lessmarked effect on the CMR fraction (measured as RP concentrations).

In terms of the public health implications of effects of exercise on postprandial lipaemia, studies which investigate moderate, easily-attainable levels of exercise appear to be warranted. Thus Hardman and her group (Aldred et al. 1994, 1995; Hardman \& Aldred, 1995) have investigated the effects of brisk walking. They showed that brisk walking after a meal reduces alimentary lipaemia (Hardman \& Aldred, 1995), moreover a single bout of brisk walking in the afternoon significantly reduced the postprandial TAG response to a test meal taken the following morning (Aldred et al. 1994). In another study they found that there was an improvement in endurance fitness when sedentary middle-aged women were trained by brisk walking over a period of 12 weeks, but that there was no effect on postprandial peak TAG levels or AUC for the postprandial TAG response to a standard test meal at the end of the training period (Aldred et al. 1995).

Only one study has examined the apo B-48 responses in relation to exercise. This study also aimed to look at the effects of a relatively-low level of exercise by comparing the lipaemic response in young male subjects who exercise three to four times per week with the responses of matched sedentary subjects (Isherwood et al. 1994). The subjects were given each of three test meals (containing 20,40 or $80 \mathrm{~g}$ fat) on different occasions. The active group had significantly lower postprandial responses (peak and AUC) after each test meal for each variable measured when compared with the inactive group. Fig. 1 shows the results for TAG and apo B-48 in a CM-enriched fraction isolated by ultracentrifugation after a $40 \mathrm{~g}$ fat meal. Interestingly, the effects of meal fat content on the apo B-48 responses was different in the two groups. The AUC for CM-TAG and CM-apo B-48 after each of the meals are shown in Fig. 2. The CM-TAG AUC increased with increasing fat content in both groups. The CM-apo B-48 AUC also increased in the inactive group with increasing fat content, but the CM-apo B-48 AUC remained the same in the active group despite the four-fold increase in meal fat content. This work shows that the potentially-beneficial lipidlowering effects of exercise are apparent even at a young age and the difference in apo B48 responses warrants further investigation to determine the mechanism behind this potentially beneficial adaptation. 

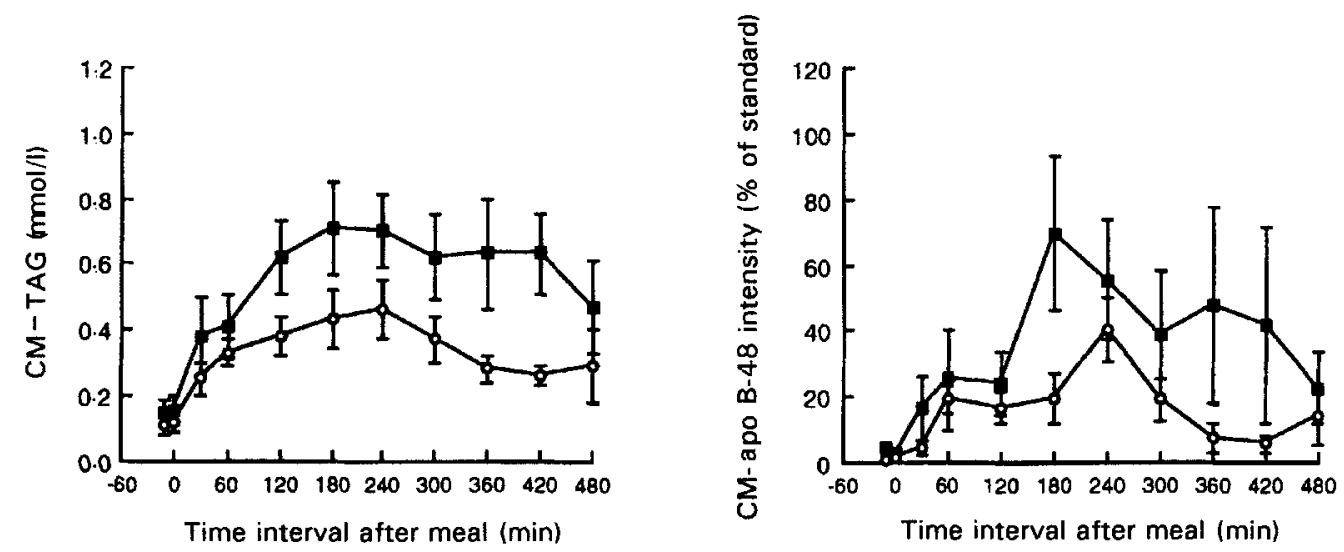

Fig. 1. Chylomicron (CM)-triacylglycerol (TAG) and CM-apolipoprotein (apo) B-48 responses in active (O) and inactive (G) subjects after a mixed meal containing $40 \mathrm{~g}$ fat. The subjects consumed the meal after a $12 \mathrm{~h}$ overnight fast and rested quietly during the $8 \mathrm{~h}$ study period. Values represent means with their standard errors represented by vertical bars for seven subjects in each group. The difference between the areas under the curves for the two groups for the CMTAG was significant $(P<0.05)$.
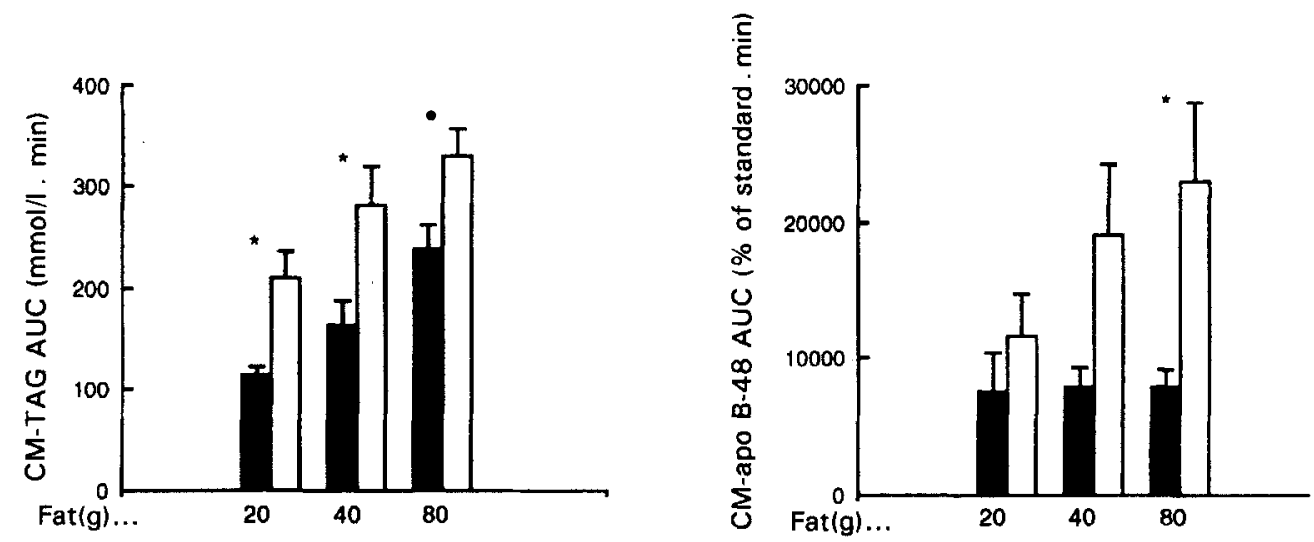

Fig. 2. Area under the curve (AUC) for chylomicron (CM)-triacylglycerol (TAG) and CM-apolipoprotein (apo) B-48 responses after a mixed meal containing either 20,40 or $80 \mathrm{~g}$ fat in active ( $\square$ ) and inactive ( $\square$ ) subjects. The meals were consumed in random order on three separate occasions after an overnight fast. Blood samples were taken for $8 \mathrm{~h}$ postprandially, during which time the subjects rested quietly. Values are means with their standard errors represented by vertical bars for seven subjects in each group. The difference between the active and inactive groups was significant, $* P<0.05$.

\section{Effects of dietary factors}

Meal fat content. Various studies have shown that increasing the fat content of a meal results in an increased postprandial lipaemia (Cohen et al. 1988; Chen et al. 1992; Murphy et al. 1995). Cohen et al. (1988) showed that there was a directly proportional increase in the TAG-AUC response to meals containing 40,80 or $120 \mathrm{~g}$ fat. Conversely, the study by Murphy et al. (1995) showed that, although there was an increase in the TAG response, it 
was not directly proportional to the increase in fat content of the meals. The main difference between the studies was in the composition of the test meals. Cohen et al. (1988) used double cream, whereas Murphy et al. (1995) used a mixed meal consisting of cheese, bread, cereal, milk and orange juice. The latter study suggests that the mechanisms involved in postprandial lipid metabolism can be regulated to maintain lipid homeostasis as fat intake increases. This may partly be caused by the higher activity of LPL with increased meal fat content which was noted in this study.

Meal frequency. A high meal frequency, i.e. a nibbling-type diet, has been shown to improve glucose tolerance (Jenkins et al. 1989) and to reduce fasting cholesterol levels (Arnold et al. 1993), whereas a gorging-type diet is associated with an increased risk of CAD, diabetes and obesity (Fabry \& Tepperman, 1970). A recent study investigated whether meal frequency produced any effects on postprandial lipid metabolism which could affect the level of potentially-atherogenic dietary-derived CMR particles present in the plasma (Murphy et al. 1996). At the end of a 2-week period on a nibblingtype diet the post-heparin LPL activity levels were found to be significantly increased compared with during the subjects normal diets. Although there was no effect on the total plasma TAG levels, there was a significantly lower TAG response associated with smaller, denser lipoprotein particles after the nibbling diet compared with the normal diet (Isherwood, 1996), suggesting that there may be some effect of meal frequency on lipid metabolism.

Fatty acids. Recent interest in the effects of diet on postprandial lipaemia has focused on modifications to the fatty acid composition of either the background diet or a single test meal, with particular reference to $n-3$ polyunsaturated fatty acids (PUFA) and monounsaturated fatty acids (MUFA). Chronic intake of $n$-3 PUFA for a period of 3-6 weeks was found to reduce the postprandial lipaemic response to a test meal (Weintraub et al. 1988; Williams et al. 1992). Zampelas et al. (1994) altered the composition of individual test meals and found that consumption of a meal enriched in $n$ - 3 PUFA produced significantly lower postprandial TAG levels than consumption of a mixed-oil meal. Conversely, Harris et al. (1988) found no difference in the TAG responses after a meal rich in saturated fatty acids compared with one rich in $n-3$ PUFA.

There have been fewer studies on the effects of MUFA on postprandial lipaemia. Brouwer et al. (1993) compared the responses to infusions of olive oil and soyabean oil and found that TAG clearance rates were slower, and hepatic lipase $(E C$ 3.1.1.3) activity higher, following olive-oil infusion. The same group showed that RP concentrations in $\mathrm{CM}$, and apo B-48 concentrations in CMR were higher, but postprandial HDL levels remained constant after a meal enriched in olive oil compared with soyabean oil, suggesting an HDL-conserving effect of olive oil (de Bruin et al. 1993). A study of the acute effect of MUFA in the meal was made by Zampelas et al. (1995). Although there was no difference in the total lipaemic response after meals containing increasing amounts of MUFA, there was a difference in the pattern of response with a biphasic pattern being shown for both TAG and apo B-48 after the high-MUFA meal ( $24 \%$ of total dietary energy as MUFA). A comparison of responses between populations which consumed different MUFA levels in their background diets was made by Jackson et al. (1996). They found that the incremental apo B-48 response to a standard test meal was significantly higher in UK subjects who habitually consumed a diet containing $11 \%$ of total dietary energy as MUFA compared with Greek subjects who habitually consumed a diet containing $17 \%$ of total dietary energy as MUFA. 


\section{CONCLUSION}

In view of the link between postprandial lipaemia and CAD, investigation of lifestyle factors which may reduce the levels of potentially-atherogenic postprandial lipoproteins are of great importance in any attempt to reduce the need for drug intervention in later life. Measurement of apo B-48 appears to be the most relevant marker for dietary-derived lipoproteins, although the nature of the protein has made it difficult to analyse. The recent production and application of a specific antiserum to apo B-48 has aided the investigation of lifestyle influences on postprandial lipaemia. Regular strenuous exercise has clearly been shown to lower postprandial lipaemia, but recently more achievable levels of exercise have also been shown to have a positive effect on both postprandial TAG and apo B-48 levels. The effects of dietary factors are now under investigation and future work may enable guidelines to be established which will help lower postprandial lipaemia.

\section{REFERENCES}

Aldred, H. E., Hardman, A. E. \& Taylor, S. (1995). Influence of 12 weeks of training by brisk walking on postprandial lipemia and insulinemia in sedentary middle-aged women. Metabolism 44, 390-397.

Aldred, H. E., Perry, I. C. \& Hardman, A. E. (1994). The effect of a single bout of brisk walking on postprandial lipemia in normolipidemic young adults. Metabolism 43, 836-841.

Arnold, L. M., Ball, M. J., Duncan, A. W. \& Mann, J. (1993). Effect of isoenergetic intake of three or nine meals on plasma lipoproteins and glucose metabolism. American Journal of Clinical Nutrition 57, 446-451.

Berr, F. (1992). Characterization of chylomicron remnant clearance by retinyl palmitate label in normal humans. Journal of Lipid Research 33, 915-930.

Blaner, W. S., Obunike, J. C., Kurlandsky, S. B., Al-Haideri, M., Piantedosi, R., Deckelbaum, R. J. \& Goldberg, I. J. (1994). Lipoprotein lipase hydrolysis of retinyl ester. Journal of Biological Chemistry 269, 15559-16565.

Brouwer, C. G., de Bruin, T. W. A., Jansen, H. \& Erkelens, D. W. (1993). Different clearance of intravenously administered olive oil and soybean-oil emulsions: role of hepatic lipase. American Journal of Clinical Nutrition 57, 533-539.

Chen, Y. D. I., Skowronski, R., Coulston, A. M., Pietarinen, J., Hollenbeck, C. B. \& Reaven, G. M. (1992). Effect of acute variations in dietary fat and carbohydrate intake on retinyl ester content of intestinally derived lipoproteins. Journal of Clinical Endocrinology and Metabolism 74, 28-32.

Chung, B. H. \& Segrest, J. P. (1990). Cytotoxicity of triglyceride-rich lipoproteins: an atherogenic insult? In Hypercholesterolemia, Hypocholesterolemia, Hypertriglyceridemia, pp. 341-351 [C. L. Malmendier, P. Alaupovic and H. B. Brewer, editors]. New York: Plenum Press.

Cohen, J. C., Noakes, T. D. \& Spinnler Benade, A. J. (1988). Serum triglyceride responses to fatty meals: effects of meal fat content. American Journal of Clinical Nutrition 47, 825-827.

Cohen, J. C., Noakes, T. D. \& Spinnler Benade, A. J. (1989). Postprandial lipemia and chylomicron clearance in athletes and in sedentary men. American Journal of Clinical Nutrition 49, 443-447.

Cohn, J. S., Johnson, E. J., Millar, J. S., Cohn, S. D., Milne, R. W., Marcel, Y. L., Russell, R. M. \& Schaefer, E. J. (1993). Contribution of apoB-48 and apoB-100 triglyceride-rich lipoproteins (TRL) to postprandial increases in the plasma concentration of TRL triglycerides and retinyl esters. Journal of Lipid Research 34, 2033-2040.

de Bruin, T. W. A., Brouwer, C. B., van Linde-Sibenius Trip, M., Jansen, H. \& Erkelens, D. W. (1993). Different postprandial metabolism of olive oil and soybean oil: a possible mechanism of the high-density lipoprotein conserving effect of olive oil. American Journal of Clinical Nutrition 58, 477-483.

Fabry, P. \& Tepperman, J. (1970). Mean frequency - a possible factor in human physiology. American Journal of Clinical Nutrition 23, 1059-1068.

Floren, C.-H., Albers, J. J. \& Bierman, E. L. (1981). Uptake of chylomicron remnants causes cholesterol accumulation in cultured human arterial smooth muscle cells. Biochimica et Biophysica Acta 663, 336-349.

Ginsberg, H. N., Jones, J., Blaner, W. S., Thomas, A., Karmally, W., Fields, L., Blood, D. \& Begg, M. D. (1995). Association of postprandial triglyceride and retinyl palmitate responses with newly diagnosed exerciseinduced myocardial ischaemia in middle-aged men and women. Arteriosclerosis and Thrombosis 15, 18291838.

Groot, P. H. E., Van Stiphout, W. A. H. J., Krauss, X. H., Jansen, H., Van Tol, A., Van Ramshorst, E., Chin-On, S., Hofman, A., Cresswell, S. R. \& Havekes, L. (1991). Postprandial lipoprotein metabolism in normolipidemic men with and without coronary artery disease. Arteriosclerosis and Thrombosis 11, 653-662.

Hardman, A. E. \& Aldred, H. E. (1995). Walking in the postprandial period decreases alimentary lipaemia. Journal of Cardiovascular Risk 2, 71-78. 
Harris, W. S., Connor, W. E., Alam, N. \& Illingworth, D. R. (1988). Reduction of postprandial triglyceridemia in humans by dietary $n-3$ fatty acids. Journal of Lipid Research 29, 1451-1460.

Isherwood, S. G. (1996). Apolipoprotein B-48 as a marker for chylomicrons and their remnants; studies in the postprandial state. PhD Thesis, University of Surrey.

Isherwood, S. G., Sethi, S., Gould, B. J., Howland, R. J. \& Williams, C. M. (1994). The effects of exercise on postprandial lipaemia: apo B-48 and retinyl palmitate as markers of chylomicron particles. Proceedings of the Nutrition Society 53, 163A.

Isherwood, S. G., Williams, C. M., Griffin, B. A. \& Gould, B. J. (1996). Potentially atherogenic chylomicron remnants are present in the low-density-lipoprotein fraction as detected by a specific antibody to apolipoprotein B-48. Proceedings of the Nutrition Society 55, 113A.

Jackson, K. G., Lovegrove, J. A., Zampelas, A., Kafatos, A., Kapsokefalou, M., Williams, C. M. \& Gould, B. J. (1996). Apolipoprotein B-48 and retinyl ester responses to meals of varying monounsaturated fatty acid contents. Proceedings of the Nutrition Society 55, 112A.

Jenkins, D. J. A., Wolever, T. M. S., Vuskan, V., Brighenti, F., Cunnane, S. C., Rao, A. V., Jenkins, A. L., Buckley, G., Patten, R., Singer, W., Corey, P. \& Josse, R. G. (1989). Nibbling versus gorging: metabolic advantages of increased meal frequency. New England Journal of Medicine 321, 929-934.

Kane, J. P., Hardman, D. A. \& Paulus, H. E. (1980). Heterogeneity of apolipoprotein B: isolation of a new species from human chylomicrons. Proceedings of the National Academy of Sciences USA 77, 2465-2469.

Karpe, F. \& Hamsten, A. (1994). Determination of apolipoproteins B-48 and B-100 in triglyceride-rich lipoproteins by analytical SDS-PAGE. Journal of Lipid Research 35, 1311-1317.

Karpe, F. \& Hultin, M. (1995). Endogenous triglyceride-rich lipoproteins accumulate in rat plasma when competing with a chylomicron-like triglyceride emulsion for a common lipolytic pathway. Journal of Lipid Research 36, 1557-1566.

Karpe, F., Steiner, G., Uffelman, K., Olivecrona, T. \& Hamsten, A. (1994). Postprandial lipoproteins and progression of coronary atherosclerosis. Atherosclerosis 106, 83-97.

Kotite, L., Bergeron, N. \& Havel, R. J. (1995). Quantification of apolipoproteins B-100, B-48, and E in human triglyceride-rich lipoproteins. Journal of Lipid Research 36, 890-900.

Krasinski, S. D., Cohn, J. S., Russell, R. M. \& Schaefer, E. J. (1990). Postprandial plasma vitamin A metabolism in humans: a reassessment of the use of plasma retinyl esters as markers for intestinally derived chylomicrons and their remnants. Metabolism 39, 357-365.

Lovegrove, J. A., Isherwood, S. G., Jackson, K. G., Williams, C. M. \& Gould, B. J. (1996). Quantitation of apolipoprotein B-48 in triacylglycerol-rich lipoproteins by a specific enzyme-linked immunosorbent assay. Biochimica et Biophysica Acta 1301, 221-229.

Mamo, J. C. L. \& Wheeler, J. R. (1994). Chylomicrons or their remnants penetrate rabbit thoracic aorta as efficiently as do smaller macromolecules, including low density lipoprotein, high density lipoprotein, and albumin. Coronary Heart Disease 5, 695-705.

Mankowitz, K., Seip, R., Semenkovich, C. F., Daugherty, A. \& Schonfeld, G. (1992). Short-term interruption of training affects both fasting and post-prandial lipoproteins. Atherosclerosis $95,181-189$.

Merrill, J. R., Holly, R. G., Anderson, R. L., Rifai, N., King, M. E. \& DeMeersman, R. (1989). Hyperlipemic response of young trained and untrained men after a high fat meal. Arteriosclerosis 9, 217-223.

Miesenbock, G. \& Patsch, J. R. (1991). Coronary artery disease: synergy of triglyceride-rich lipoproteins and HDL. Cardiovascular Risk Factors 1, 293-299.

Murphy, M. C., Chapman, C., Lovegrove, J. A., Isherwood, S. G., Morgan, L. M., Wright, J. W. \& Williams, C. M. (1996). Meal frequency: does it determine postprandial lipaemia? European Journal of Clinical Nutrition 50, $491-497$.

Murphy, M. C., Isherwood, S. G., Sethi, S., Gould, B. J., Wright, J. W., Knapper, J. M. E. \& Williams, C. M. (1995). Postprandial lipid and hormone responses to meals of varying fat contents: modulatory role of lipoprotein lipase? European Journal of Clinical Nutrition 49, 579-588.

Patsch, J. R., Karlin, J. B., Scott, L. W., Smith, L. C. \& Gotto, A. M. Jr (1983). Inverse relationship between blood levels of high density lipoprotein subfraction 2 and magnitude of postprandial lipemia. Proceedings of the National Academy of Sciences USA 80, 1449-1453.

Peel, A. S., Komanduri, P., Williams, C. M., Morgan, L. M. \& Gould, B. J. (1992). A specific antibody to apolipoprotein B-48: a novel approach. Biochemical Society Transactions 20, 165 S.

Peel, A. S., Zampelas, A., Williams, C. M. \& Gould, B. J. (1993). A novel antiserum specific to apolipoprotein B-48: application in the investigation of postprandial lipidaemia in humans. Clinical Science 85, 521-524.

Podl, T. R., Zmuda, J. M., Yurgalevitch, S. M., Fahrenbach, M. C., Bausserman, L. L., Terry, R. B. \& Thompson, P. D. (1994). Lipoprotein lipase activity and plasma triglyceride clearance are elevated in endurance-trained women. Metabolism 43, 808-813.

Rapp, J. H., Lespine, A., Hamilton, R. L., Colyvas, N., Chaumeton, A. H., Tweedie-Hardman, J., Kotite, L., Kunitake, S. T., Havel, R. J. \& Kane, J. P. (1994). Triglyceride-rich lipoproteins isolated by selected-affinity anti-apolipoprotein B immunosorption from human atherosclerotic plaque. Arteriosclerosis and Thrombosis 14, 1767-1774. 
Schneeman, B. O., Kotite, L., Todd, K. M. \& Havel, R. J. (1993). Relationships between the responses of triglyceride-rich lipoproteins in blood plasma containing apolipoproteins B-48 and B-100 to a fat-containing meal in normolipidemic humans. Proceedings of the National Academy of Sciences USA 90, 2069-2073.

Simpson, H. S., Williamson, C. M., Olivecrona, T., Pringle, S., Maclean, J., Lorimer, A. R., Bonnefous, F., Bogaievsky, Y., Packard, C. J. \& Shepherd, J. (1990). Postprandial lipemia, fenofibrate and coronary artery disease. Atherosclerosis 85, 193-202.

Tall, A. R. (1993). Plasma cholesteryl ester transfer protein. Joumal of Lipid Research 34, 1255-1274.

Uiterwaal, C., Grobbee, D., Witteman, J., van Stiphout, W., Krauss, X., Havekas, L., de Bruijn, A., van Tol, A. \& Hofman, A. (1994). Postprandial triglyceride response in young adult men and familial risk from coronary atherosclerosis. Annals of Internal Medicine 121, 576-583.

Weintraub, M. S., Eisenberg, S. \& Breslow, J. L. (1987). Different patterns of postprandial lipoprotein metabolism in normal, type IIa, type III, and type IV hyperlipoproteinemic individuals. Journal of Clinical Investigation 79, 1110-1119.

Weintraub, M. S., Rosen, Y., Otto, R., Eisenberg, S. \& Breslow, J. L. (1989). Physical exercise conditioning in the absence of weight loss reduces fasting and postprandial triglyceride-rich lipoprotein levels. Circulation 79 , 1007-1014.

Weintraub, M. S., Zechner, R., Brown, A., Eisenberg, S. \& Breslow, J. L. (1988). Dietary polyunsaturated fats of the $\omega-6$ and $\omega-3$ series reduce postprandial lipoprotein levels. Journal of Clinical Investigation 82, 1884 1893.

Williams, C. M., Moore, F., Morgan, L. M. \& Wright, J. (1992). Effects of $n-3$ fatty acids on postprandial triacylglycerol and hormone concentrations in normal subjects. British Journal of Nutrition 69, 63-78.

Young, S. G. (1990). Recent progress in understanding apolipoprotein B. Circulation 82, 1574-1594.

Zampelas, A., Knapper, J. M. E., Jackson, K. G., Culverwell, C. C., Wilson, J., Gould, B. J. \& Williams, C. M. (1995). Postprandial triacylglycerol and apolipoprotein B-48 responses to meals of varying monounsaturated fatty acid content in young UK subjects. Atherosclerosis 115, Suppl., S46.

Zampelas, A., Peel, A. S., Gould, B. J., Wright, J. \& Williams, C. M. (1994). Polyunsaturated fatty acids of the $n-6$ and $n-3$ series: effects on postprandial lipid and apolipoprotein levels in healthy men. European Journal of Clinical Nutrition 48, 842-848.

Zilversmit, D. B. (1979). Atherogenesis: a postprandial phenomenon. Circulation 60, 473-485. 